\section{Prevalência e fatores associados à percepção negativa da saúde em pessoas idosas no Brasil}

\section{Prevalence and factors associated with negative health perception by the Brazilian elderly}

\author{
Roberto Jerônimo dos Santos Silva ${ }^{1, I I}$ \\ Aldemir Smith-Menezes, ${ }^{1, I I}$ \\ Sheilla Tribess ${ }^{\mathrm{IV}}$ \\ Vicente Rómo-Perez ${ }^{1, v}$ \\ Jair Sindra Virtuoso Júnior'v \\ 'Pós graduación en Actividad Física y Salud de la Facultade de Ciencias de \\ Educación y del Deporte - UVIGO, Pontevedra, España. \\ "Departamento de Educação Física da Universidade Federal de Sergipe - UFS. \\ "'Instituto Federal de Educação, Ciência e Tecnologia de Sergipe - IFS, Campus \\ Itabaiana, SE. \\ IV Instituto de Ciências da Saúde da Universidade Federal do Triângulo Mineiro - \\ UFTM, Uberaba, MG. \\ ` Departamento de Didácticas Especiais da Facultade de Ciencias de Educación \\ y del Deporte - UVIGO, Pontevedra, España.
}

Correspondência: Roberto Jerônimos dos Santos Silva. Departamento de Educação Física/Universidade Federal de Sergipe. Av. Marechal Rondon S/N - Jd. Rosa Elze - São Cristovão - CEP 49100000. E-mail: rjeronimoss@gmail.com

\section{Resumo}

Objetivo: Analisar os fatores associados à percepção negativa de saúde em idosos brasileiros. Métodos: Estudo de base populacional com delineamento transversal realizado no ano de 2009, em três cidades do Brasil. A amostra estratificada de forma aleatória simples foi composta de 909 adultos de 60 a 91 anos. Na identificação dos fatores associados com a percepção negativa de saúde (PNS), foi realizada análise bruta e mutivariável por estimativas das Razões de Prevalência (RP), por meio da regressão de Poisson; $\mathrm{p}<0.05$. Resultados: A prevalência para PNS foi de $49,6 \%$, e após análise multivariada foi observado associação para os homens com o aumento da idade RP = 1,03 (IC = 1,01 - 1,06), risco de desnutrição $\mathrm{RP}=1,66(\mathrm{IC}=1,13-2,43)$ e incapacidade funcional $\mathrm{RP}=1,79$ (IC = 1,21-1,77). Para as mulheres, o uso superior a dois medicamentos RP = 1,41 (IC = 1,13 - 2,52), hipertensão arterial $\mathrm{RP}=1,52(\mathrm{IC}=1,43-1,97)$ e a incapacidade funcional $\mathrm{RP}=1,36$ (IC = 1,13 - 1,86). Conclusões: Quase a metade dos idosos possuem uma PNS, de maneira que ações preventivas de diminuição ao uso de medicamentos e comportamentos que favoreçam melhores condições nutricionais devem estar atreladas às políticas públicas à promoção da autonomia funcional e do bem-estar dos idosos.

Palavras-chave: Saúde do idoso. Autoavaliação (saúde). Autonomia funcional. Bem-estar. Percepção de saúde. idoso. 


\section{Abstract}

Objective: To analyze the factors associated with negative health perception in older Brazilians. Methods: Population study of a cross-sectional sample, conducted in 2009 in three cities of Brazil. The stratified simple random sample consisted of 909 adults between 60 and 91 years of age. A gross and multivariate analysis by estimated prevalence rate (PR) using Poisson regression with calculation of adjusted prevalence ratios, $p$ $<0.05$ was performed to identify factors associated with negative perception of health (NPH). Results: The prevalence of NPH was $49.6 \%$. After the multivariate association, a certain association for men with older age $\mathrm{PR}=1.03(\mathrm{Cl}=1.01$ to 1.06$)$, risk of malnutrition $\mathrm{PR}=1.66(\mathrm{CI}=1.13$ to 2.43$)$ and disability $\mathrm{PR}=1.79(\mathrm{CI}=1.21$ to 1.77$)$ was observed. For women, the association was with taking more than two medications $\mathrm{PR}=1.41(\mathrm{CI}=$ 1.13 to 2.52), hypertension $\mathrm{PR}=1.52(\mathrm{CI}=$ 1.43 to 1.97 ) and functional disability $\mathrm{PR}=$ $1.36(\mathrm{CI}=1.13$ to 1.86$)$. Conclusions: Nearly half of the elderly have NHP, therefore preventive actions that decrease drug use and encourage behaviors toward better nutritional conditions should be tied to public policies in order to promote the functional independence and well-being of the elderly.

Keywords: Elderly health. Self sssessment in health. Functional autonomy. Wellness. Health perception. Elderly.

\section{Introdução}

A efetividade na avaliação do estado de saúde é uma das preocupações quando se procura avaliar as condições de saúde em idosos. Esta avaliação, quando se dispõe de local e equipamentos adequados, não se configura como problema; no entanto, os métodos clínicos e laboratoriais geralmente utilizados para a avaliação do estado de saúde em grupos populacionais podem se configurar como procedimentos inviáveis.

Outro ponto interessante trata da natureza subjetiva da auto-avaliação; de modo geral, os estudos de cunho epidemiológico terminam por não esclarecer quais elementos são considerados pelos idosos no que se refere ao seu estado de saúde ${ }^{1}$.

Neste sentido, torna-se necessária a elaboração de estratégias simples, de fácil compreensão e baixo custo financeiro, de maneira a favorecer a eficácia em diagnósticos, sobretudo em inquéritos populacionais. Dentre as opções para avaliação do estado de saúde, as medidas subjetivas, por intermédio de escalas (questionários), parecem ser a estratégia mais viável em estudos populacionais em virtude de sua praticidade.

Nesta mesma linha de raciocínio, verifica-se que a percepção subjetiva de saúde se configura como a interpretação pessoal que os indivíduos fazem de sua própria saúde ${ }^{1,2}$. Tal percepção está diretamente dependente das experiências pessoais, dos objetivos e dos mecanismos que as pessoas utilizam para lidar com decepções e fracassos, podendo ainda estar relacionada com as condições físicas e com o comprometimento funcional do sujeito ${ }^{2,3}$.

A auto-avaliação subjetiva do estado de saúde tem merecido considerável atenção como um indicador associado ao declínio da autonomia funcional e até mesmo da mortalidade entre idosos ${ }^{3,4,5}$. Tem sido largamente utilizada por ser uma variável simples de ser obtida, com o potencial de sintetizar uma complexa interação de fatores envolvidos na saúde do idoso, e com valor preditivo de mortalidade $\mathrm{e}^{3,5}$. 
Embora haja diversos estudos evidenciando a associação entre medidas de saúde objetivas e medidas auto-referidas de saúde, poucos se preocuparam em verificar a relação da percepção de saúde com a interação de diversos fatores (sociodemográficos e econômicos, capacidade funcional e com problemas de saúde $)^{4,5}$.

A análise dos fatores relacionados à auto-percepção de saúde estratificada por sexo permite identificar com mais detalhes as variáveis que interferem na saúde dos idosos, pois o processo de envelhecimento é marcado por diferenças no declínio físico e metabólico de homens e mulheres ${ }^{6}$, fato que pode desencadear percepções diferenciadas em torno da saúde.

O propósito deste estudo foi verificar, em idosos, a relação da percepção de saúde com aspectos sociodemográficos e econômicos, aspectos clínicos e comportamentais. O levantamento dessas informações proporciona o entendimento do papel da auto-avaliação do estado de saúde, servindo como referência para fundamentar programas na promoção à saúde no diagnóstico e acompanhamento de possíveis intervenções que venham a ser desenvolvidas a grupamentos etários mais avançados.

\section{Métodos}

Foi realizado um estudo transversal, com inquérito domiciliar, em três municípios interioranos brasileiros representando as regiões Nordeste, Sudeste e Sul do país (respectivamente, Distrito de Olivença em Ilhéus/BA, Caratinga/MG e Nova Santa Rosa/PR), no período de março a maio de 2009. A amostra populacional, foi calculada em 992 idosos (Caratinga $=463$; Ilhéus = 148; Nova Santa Rosa $=298$ ), considerando $95 \%$ de confiança, efeito desing 2,5, $80 \%$ de poder do teste, margem de erro (me) de $5,0 \%$ para as estimativas intervalares e uma proporção estimada de $n=0,5$ para as proporções de interesse? ${ }^{7}$.

Para o cálculo dos idosos, em cada município foi respeitada a proporção populacional, sendo que para o cálculo dos idosos nos bairros elegíveis, a partir do procedimento de sorteio, em cada município utilizou-se a técnica de amostragem estratitificada proporcional, que consta dos seguintes procedimentos na ordem de ocorrência:

- conhecimento do número de idosos em cada bairro com a utilização dos dados disponibilizados pelo Ministério da Saúde - Secretaria Municipal de Saúde nos respectivos municípios;

- cálculo da proporção que cada estrato (bairro) representa na população $\left(\mathrm{p}_{\mathrm{i}}\right)$ onde $\mathrm{N}=$ número total de idosos nos municípios; $\mathrm{N}_{\mathrm{i}}=$ número total de idosos em cada estrato (bairro) ${ }^{7}$;

- cálculo do número de sujeitos da amostra em cada estrato $\left(\mathrm{n}_{\mathrm{i}}\right)$; onde " $\mathrm{n}$ " representa o número de idosos da amostra; pi: proporção que cada estrato representa na população: $\mathrm{n}_{\mathrm{i}}=\mathrm{p}_{\mathrm{i}}{ }^{*} \mathrm{n}$

Após o cálculo do número de idosos para a amostra em cada estrato (bairro ou distrito), foram considerados pelo menos 10 idosos naqueles setores, onde o cálculo amostral foi inferior a cinco. Utilizou-se a técnica de amostra sistemática para selecionar, dentro de cada bairro, os domicílios, nos quais os idosos foram entrevistados; portanto, a amostra inicial foi de 992 idosos. Com as perdas previstas (10\%), obteve-se uma amostra final de 909 idosos.

Para a seleção dos idosos a serem entrevistados em cada bairro, após o cálculo do intervalo amostral realizou-se sorteio aleatório para selecionar o primeiro domicílio a ser visitado na primeira quadra do bairro. No sentido horário, os demais domicílios foram considerados em cada quadra, até percurso da totalidade do bairro. Nos domicílios em que não havia idoso, considerou-se aquele imediatamente posterior. Realizou-se a escolha por sorteio quando, na residência selecionada, havia mais de um idoso. Quando no domicílio selecionado havia idoso, mas este não estava em casa, agendou-se outro horário, e se no dia agendado o idoso estivesse ausente, considerou-se o domicílio imediatamente 
posterior. Em condomínios (prédios ou casas), o entrevistador apresentou-se na portaria e questionou sobre o número de apartamentos ou casas existentes, elaborou um roteiro e deu sequência à seleção dos domicílios.

Utilizou-se o mapa de cada bairro disponibilizado pelas Secretarias Municipais de Saúde como itinerário para a seleção dos domicílios. Quando o bairro foi percorrido e não se alcançou o número de idosos desejado, voltou-se ao início do bairro, na casa subsequente à primeira casa selecionada $\mathrm{e}$ todos os procedimentos foram repetidos, até se obter o número desejado de idosos.

Para o presente estudo, a amostra populacional foi constituída por idosos que atenderam aos critérios de inclusão: ter 60 anos ou mais, residir na Zona Urbana dos municípios investigados e nos respectivos bairros ou distrito selecionados, não apresentar sintomatologia de confusão mental acentuada e aceitar participar da pesquisa.

Para a coleta de dados foi utilizada uma entrevista multidimensional, aplicada individualmente. Deve-se ressaltar que os instrumentos tiveram sua confiabilidade testada previamente ao estudo, constituído pelos seguintes itens:

- Dimensão sociodemográfica: idade, sexo, cor da pele, escolaridade (último grau completo), estado civil, atividade laboral, tamanho da família e nível socioeconômico (adotado o Critério Brasil de Classificação Econômica)*;

- Dimensão de saúde física e mental: autopercepção de saúde, consumo de bebidas alcoólicas e tabagismo, patologias em conformidade com a Classificação Internacional de Doenças (CID-10) ${ }^{8}$, risco de desnutrição pela Mini avaliação nutricional ${ }^{9}$, e atividades instrumentais da vida diária (AIVD) pela versão adaptada do Índice de Lawton ${ }^{10}$. Adotou-se o ponto de corte de 12 pontos para a presença de dependência funcional do tipo moderada a grave, e sintomatologia depressiva avaliada por intermédio da Escala de Depressão Geriátrica (GDS$15)^{11}$, com o ponto de corte padrão de cinco pontos para a presença de sintomatologia depressiva;

- Aspectos de saúde mental: na avaliação da função cognitiva foi utilizado o Questionário Brazil Old Age Schedule $(\mathrm{BOAS})^{* *}$, com ponto de corte padrão de quatro pontos para a presença de alterações cognitivas severas e nível de atividade física habitual por por intermédio da versão longa do Questionário Internacional de Atividade Física (IPAQ), adaptada para idosos ${ }^{12}$.

A variável dependente foi representada pela percepção negativa de saúde, constando dos idosos que referiram a saúde como regular ou ruim.

Para confecção do banco de dados foi utilizado o software Epidata, versão 3.1b, e as análises por meio do pacote estatístico SPSS (versão 15). De forma a extrair melhores informações da abordagem descritiva, foi realizada a análise de associação do qui-quadrado. Para a identificação dos fatores associados com a percepção negativa de saúde, foi realizada análise bruta e mutivariável por meio da regressão de Poisson. Todas as variáveis foram para análise multivariável independente do $\mathrm{p}$ valor na análise bruta, seguindo o modelo teórico de determinação. Para escolha da sequência de seleção das variáveis de entrada no modelo multivariável foi utilizado o método "backward".

Mediante a estratégia estabelecida de associações entre as dimensões estudadas, foram elaborados três modelos explicativos utilizando-se a regressão de Poisson e introduzindo as variáveis em forma de blocos, conforme segue: No bloco 1 estão as variáveis sexo, cor da pele e idade; no bloco 2 , as variáveis cidade, estado civil, escolaridade, religião, condição socioeconômica,

*ABEP. Critério de Classificação Econômica Brasil 2003. Disponível em http:www.abep.org/codigosguias/ABEP_CCEB_2003 [Acessado em 20 de março de 2003]

**Veras R, Dutra S. Perfil do idoso brasileiro - questionário BOAS. Rio de Janeiro: UERJ , UnATI; 2008. 100 p. Disponível em: http://www. crde-unati.uerj.br/liv_pdf/perfil.pdf. [Acessado em 8 de julho de 2008] 
ocupação, fonte atual de renda e rendimento mensal; no bloco 3 , as variáveis nutrição, uso atual de fumo, consumo atual de álcool, atividade física regular no passado, avaliação cognitiva, sintomatologia depressiva e capacidade funcional na AIVD. As variáveis permaneceram no modelo quando o $\mathrm{p}$ valor do teste de wald foi $\leq 0,20$. Considerou-se um nível de significância $\mathrm{p}<0,05$ e intervalo de confiança (IC) de $95 \%$, com cálculo das razões de prevalências ajustadas.

Esta pesquisa seguiu os princípios éticos presentes na Declaração de Helsinque. Os protocolos de pesquisa foram avaliados $\mathrm{e}$ aprovados pelo Comitê de Ética em Pesquisa com Seres Humanos da Universidade Estadual de Santa Cruz, Brasil, (Parecer $\mathrm{n}^{\circ}$. 32/08).

\section{Resultados}

Participaram deste estudo 909 idosos (taxa de resposta de 91,63\%). O elevado grau de déficit cognitivo e o não consentimento em participar do estudo foram os principais motivos das perdas amostrais.

A frequência de idosos que referiram a percepção negativa de saúde foi de $49,6 \%$, sendo maior no sexo feminino $(56,7 \%)$ do que no masculino (43,3\%). A média de idade foi de 71,47 anos (DP = 8,01), com amplitude de 60 a 91 anos.

A Tabela 1 descreve as características dos idosos por condição de saúde referida. Mais da metade da população foi classificada como de cor de pele branca; entretanto, não houve diferenças significativas na percepção de saúde entre os sexos. De forma similar, o estado civil não interferiu para as diferenças entre os sexos em relação à percepção de saúde negativa. A percepção de saúde negativa foi diferenciada para os sexos quando analisado o município.

A maioria dos idosos apresentou escolaridade até o ginasial completo (ensino fundamental), eram católicos e das classes econômicas C, D e E. No entanto, as diferenças na percepção de saúde negativa para os sexos foram observadas somente para classe econômica. Cerca de 3/4 dos idosos eram aposentados, grupo que também se apresentou com elevada frequência de percepção negativa de saúde, sendo as prevalências diferenciadas para os sexos.

Também foi observado que cerca de dois terços dos idosos são classificados, quanto ao nível nutricional, como "normal"; além disso, observa-se uma relação do sexo com a percepção negativa de saúde. As prevalências de tabagismo e de consumo de álcool para aqueles que declaram percepção de saúde negativa foi diferenciada por sexo. Aproximadamente três quartos da amostra não consome álcool e não faz uso do fumo na atualidade.

Cerca de dois terços das pessoas não praticou atividade física no passado, sendo desse grupo as mulheres mais pessimistas em relação à saúde quando comparadas aos homens. Entre os inativos, 83\% referiram a percepção negativa de saúde. Claramente, a maioria da amostra não apresenta alterações cognitivas do tipo "moderada" ou "severa" e "sintomatologia depressiva". Por fim, as idosas com incapacidade funcional mostraram-se menos otimistas com relação ao estado de saúde.

As Tabelas 2 e 3 apresentam a análise bruta entre a percepção negativa da saúde e as variáveis independentes e análise ajustada, para as variáveis com $\mathrm{p}<0,20$, separadamente entre os sexos. Observando estas tabelas percebe-se que, em relação à variável "idade", para ambos os sexos a média mais elevada de idade se apresentou associada à percepção negativa de saúde.

Entre as idosas, o baixo estrato econômico, e renda familiar, a inatividade física, a desnutrição, uso de medicamentos, problemas cardíacos, hipertensão arterial, diabetes mellitus, sintomatologia depressiva e incapacidade funcional apresentaram uma tendência de associação na análise bruta. Para o sexo masculino, algumas variáveis se comportaram diferentemente, sendo que a condição de viuvez, a baixa escolaridade, baixa renda familiar, inatividade física, estado de desnutrição, uso de dois ou mais medicamentos, problemas cardíacos, hipertensão arterial, diabetes mellitus, déficit 
Tabela 1 - Características sociodemográficas e comportamentais associadas a percepção de saúde negativa em idosos. Brasil, 2008.

Table 1 - Sociodemographic and behavioral characteristics associated with the negative perception of health in the elderly. Brazil, 2008.

\begin{tabular}{|c|c|c|c|c|}
\hline \multirow[t]{3}{*}{ Variáveis } & \multicolumn{4}{|c|}{ Percepção da Saúde Negativa } \\
\hline & \multicolumn{2}{|r|}{$\begin{array}{c}\text { Mulheres } \\
(n=295)\end{array}$} & $\begin{array}{l}\text { Homens } \\
(n=156)\end{array}$ & \multirow[b]{2}{*}{ p-valor* } \\
\hline & $\mathrm{n}$ & $\%$ & $\%$ & \\
\hline \multicolumn{5}{|l|}{ Cor da Pele } \\
\hline Branca & 237 & 49,5 & 59,0 & 0,234 \\
\hline Parda & 145 & 34,8 & 27,6 & \\
\hline Negra & 67 & 15,7 & 13,5 & \\
\hline \multicolumn{5}{|l|}{ Estado Civil } \\
\hline Solteiro & 20 & 4,1 & 5,2 & 0,276 \\
\hline Casado & 233 & 49,2 & 56,8 & \\
\hline Viúva & 173 & 41,7 & 32,3 & \\
\hline Divorciado & 24 & 5,1 & 5,8 & \\
\hline \multicolumn{5}{|l|}{ Cidade } \\
\hline Caratinga & 212 & 47,5 & 47,4 & 0,026 \\
\hline Ilhéus & 77 & 20,3 & 11,2 & \\
\hline Nova Santa Rosa & 158 & 32,2 & 41,4 & \\
\hline \multicolumn{5}{|l|}{ Escolaridade } \\
\hline Analfabeto/Primário incompleto & 313 & 69,4 & 69,9 & 0,544 \\
\hline Primário completo/Ginasial incompleto & 116 & 25,2 & 26,9 & \\
\hline Ginasial completo & 21 & 5,4 & 3,2 & \\
\hline \multicolumn{5}{|l|}{ Religião } \\
\hline Católico & 291 & 66,1 & 61,5 & 0,286 \\
\hline Protestante ou Evangélica & 106 & 21,0 & 28,2 & \\
\hline Outros & 47 & 11,5 & 8,3 & \\
\hline Nenhuma & 07 & 1,4 & 1,9 & \\
\hline \multicolumn{5}{|l|}{ Condição Socioeconômica } \\
\hline Classe $A / B$ & 37 & 7,1 & 10,3 & 0,045 \\
\hline Classe C & 208 & 43,1 & 51,9 & \\
\hline Classe D/E & 206 & 49,8 & 37,8 & \\
\hline \multicolumn{5}{|l|}{ Ocupação } \\
\hline Aposentado, mas trabalha & 17 & 2,7 & 5,8 & $<0,001$ \\
\hline Só aposentado & 357 & 75,9 & 85,3 & \\
\hline Só dona de casa & 33 & 10,2 & 1,9 & \\
\hline Pensionista & 30 & 9,5 & 1,3 & \\
\hline Trabalho Remunerado & 14 & 1,7 & 5,8 & \\
\hline \multicolumn{5}{|l|}{ Fonte de Renda Atual } \\
\hline Trabalho & 25 & 3,4 & 9,6 & 0,002 \\
\hline Previdência /aposentadoria & 387 & 85,4 & 86,5 & \\
\hline Bolsa Família & 03 & 1,0 & 0 & \\
\hline Não Possui Renda & 16 & 5,4 & 0 & \\
\hline Outros & 20 & 4,7 & 3,8 & \\
\hline
\end{tabular}


Tabela 1 - Características sociodemográficas e comportamentais associadas a percepção de saúde negativa em idosos. Brasil, 2008. (continuação)

Table 1 - Sociodemographic and behavioral characteristics associated with the negative perception of health in the elderly. Brazil, 2008. (continuation)

\begin{tabular}{|c|c|c|c|c|}
\hline \multirow[t]{3}{*}{ Variáveis } & \multicolumn{4}{|c|}{ Percepção da Saúde Negativa } \\
\hline & \multicolumn{2}{|r|}{$\begin{array}{l}\text { Mulheres } \\
(\mathrm{n}=295)\end{array}$} & $\begin{array}{c}\text { Homens } \\
(n=156)\end{array}$ & \multirow[b]{2}{*}{$\mathrm{p}$-valor* } \\
\hline & $\mathrm{n}$ & $\%$ & $\%$ & \\
\hline \multicolumn{5}{|l|}{ Nutrição } \\
\hline Desnutrido & 23 & 4,7 & 6,5 & 0,493 \\
\hline Risco de Desnutrição & 113 & 27,7 & 23,4 & \\
\hline Normal & 296 & 67,6 & 70,1 & \\
\hline \multicolumn{5}{|l|}{ Consumo Atual de Álcool } \\
\hline Consome & 41 & 7,1 & 12.8 & 0,045 \\
\hline Não Consome & 410 & 92,9 & 87,2 & \\
\hline \multicolumn{5}{|l|}{ Uso do Fumo Atual } \\
\hline Faz Uso & 42 & 5,4 & 16,7 & $<0,001$ \\
\hline Não Faz Uso & 409 & 94,6 & 83,3 & \\
\hline \multicolumn{5}{|l|}{ Atividade Física no Passado } \\
\hline Praticou & 122 & 23,5 & 34,2 & 0,015 \\
\hline Não Praticou & 327 & 76,5 & 65,8 & \\
\hline \multicolumn{5}{|l|}{ Atividade Física Atual } \\
\hline Pratica & 76 & 17,6 & 16,0 & 0,676 \\
\hline Não Pratica & 370 & 82,4 & 84,0 & \\
\hline \multicolumn{5}{|l|}{ Alterações Cognitivas } \\
\hline Sem alterações & 401 & 89,5 & 89,0 & 0,863 \\
\hline Com alterações & 48 & 10,5 & 11,0 & \\
\hline \multicolumn{5}{|l|}{ Sintomatologia Depressiva } \\
\hline Sem sintomas & 381 & 80,3 & 92,3 & 0,001 \\
\hline Com sintomas & 70 & 19,7 & 7,7 & \\
\hline \multicolumn{5}{|l|}{ Capacidade Funcional na AIVD } \\
\hline Sem Incapacidade Funcional & 211 & 48,6 & 43,6 & 0,307 \\
\hline Com Incapacidade Funcional & 239 & 51,4 & 56,4 & \\
\hline
\end{tabular}

* teste de qui-quadrado./ *chi-square test

cognitivo e a incapacidade funcional foram associados à percepção negativa da saúde.

$\mathrm{Na}$ análise ajustada, o uso de dois ou mais medicamentos, a hipertensão arterial e a incapacidade funcional permaneceram associadas à percepção de saúde negativa para as mulheres; já para os homens, o aumento da idade, a desnutrição e a incapacidade funcional foram as variáveis que permaneceram no modelo de análise ajustada.

\section{Discussão}

O estudo identificou as variáveis associadas à percepção de saúde negativa em idosos em três cidades brasileiras. Apesar da relação com algumas morbidades, a incapacidade funcional é a variável que mais interfere na percepção pessimista do estado de saúde, independentemente do sexo.

Os dados sociodemográficos descritos na Tabela 1 indicam uma distribuição 
Tabela 2 - Razão de Prevalência (RP) bruta e multivariável para varíaveis independentes em relação a percepção negativa da saúde em mulheres. Brasil, 2008.

Table 2 - Crude and multivariable Prevalence Ratio (PR) for independent variables in the negative perception of health in women. Brazil, 2008.

\begin{tabular}{|c|c|c|c|c|c|}
\hline \multirow[t]{3}{*}{ Variáveis } & \multirow[t]{3}{*}{ Prevalência } & \multicolumn{4}{|c|}{ Percepção Negativa da Saúde } \\
\hline & & \multicolumn{2}{|c|}{ Análise Bruta } & \multicolumn{2}{|c|}{ Análise Multivariável" } \\
\hline & & $\mathrm{RP}(\mathrm{IC} 95 \%)$ & Wald (p-valor) & RP (IC 95\%) & Wald ( $p$-valor) \\
\hline \multicolumn{6}{|l|}{ Cor da Pele } \\
\hline Branca & 48,3 & 1 & $3,013(0,222)$ & & \\
\hline Parda & 59,3 & $1,23(0,95-1,58)$ & & & \\
\hline Negra & 59,0 & $1,22(0,88-1,70)$ & & & \\
\hline Idade & & $1,02(1,01-1,03)$ & $4,636(0,031)$ & $1,01(1,00-1,03)$ & $1,616(0,204)$ \\
\hline Cidade & 52,6 & & & & \\
\hline Caratinga & 58,8 & 1 & $0,698(0,706)$ & & \\
\hline Ilhéus & 58,8 & $1,12(0,83-1,51)$ & & & \\
\hline Nova Santa Rosa & 51,6 & $0,98(0,76-1,27)$ & & & \\
\hline \multicolumn{6}{|l|}{ Estado Civil } \\
\hline Solteiro & 48,0 & 1 & $0,744(0,863)$ & & \\
\hline Casado & 51,6 & $1,08(0,60-1,94)$ & & & \\
\hline Viúva & 56,4 & $1,18(0,65-2,13)$ & & & \\
\hline Divorciado & 50,0 & $1,04(0,49-2,23)$ & & & \\
\hline \multicolumn{6}{|l|}{ Escolaridade } \\
\hline Analfabeto & 66,0 & 1 & $8,939(0,063)$ & 1 & $1,831(0,767)$ \\
\hline Primário Incompleto & 56,4 & $0,86(0,64-1,15)$ & & $0,94(0,69-1,29)$ & \\
\hline Primário Completo & 46,4 & $0,71(0,51-0,99)$ & & $0,85(0,59-1,22)$ & \\
\hline Ginasial Completo & 40,0 & $0,61(0,31-1,18)$ & & $0,74(0,37-1,53)$ & \\
\hline Colegial Completo & 25,0 & $0,38(0,16-0,88)$ & & $0,59(0,21-1,65)$ & \\
\hline \multicolumn{6}{|l|}{ Religião } \\
\hline Católico & 53,3 & 1 & $3,201(0,202)$ & & \\
\hline Protestante ou Evangélica & 47,0 & $0,88(0,66-1,17)$ & & & \\
\hline Outros & 67,8 & $1,27(0,90-1,80)$ & & & \\
\hline \multicolumn{6}{|l|}{ Condição Socioeconômica } \\
\hline Classe $A / B$ & 39,6 & 1 & $8,588(0,014)$ & 1 & $1,480(0,477)$ \\
\hline Classe C & 47,2 & $1,24(0,78-1,97)$ & & $0,90(0,50-1,62)$ & \\
\hline Classe D/E & 63,4 & $1,66(1,05-2,63)$ & & $1,05(0,57-1,95)$ & \\
\hline \multicolumn{6}{|l|}{ Ocupação } \\
\hline Aposentado, mas trabalha & 34,8 & 1 & $2,449(0,654)$ & & \\
\hline Só aposentado & 53,8 & $1,55(0,77-3,13)$ & & & \\
\hline Só dona de casa & 60,0 & $1,73(0,79-3,76)$ & & & \\
\hline Pensionista & 54,9 & $1,58(0,72-3,76)$ & & & \\
\hline Trabalho Remunerado & 38,5 & $1,11(0,36-3,38)$ & & & \\
\hline \multicolumn{6}{|l|}{ Fonte de Renda Atual } \\
\hline Trabalho & 38,5 & 1 & $1,204(0,877)$ & & \\
\hline Previdência /aposentadoria & 53,7 & $1,40(0,74-2,63)$ & & & \\
\hline Bolsa família & 50,0 & $1,30(0,36-4,72)$ & & & \\
\hline Não possui renda & 57,1 & $1,49(0,67-3,27)$ & & & \\
\hline Outros & 56,0 & $1,46(0,65-3,28)$ & & & \\
\hline
\end{tabular}


Tabela 2 - Razão de Prevalência (RP) bruta e multivariável para varíaveis independentes em relação a percepção negativa da saúde em mulheres. Brasil, 2008. (continuação)

Table 2 - Crude and multivariable Prevalence Ratio (PR) for independent variables in the negative perception of health in women. Brazil, 2008. (continuation)

\begin{tabular}{|c|c|c|c|c|c|}
\hline \multirow[t]{3}{*}{ Variáveis } & \multirow[t]{3}{*}{ Prevalência } & \multicolumn{4}{|c|}{ Percepção Negativa da Saúde } \\
\hline & & \multicolumn{2}{|c|}{ Análise Bruta } & \multicolumn{2}{|c|}{ Análise Multivariável $^{*}$} \\
\hline & & $\mathrm{RP}(\mathrm{IC}$ 95\%) & Wald (p-valor) & $\mathrm{RP}(\mathrm{IC} 95 \%)$ & Wald (p-valor) \\
\hline \multicolumn{6}{|l|}{ Rendimento Mensal } \\
\hline Até 2 Salários Mínimos & 59,6 & 1 & $10,457(0,005)$ & 1 & $3,872(0,144)$ \\
\hline 2-4 Salários Mínimos & 48,3 & $0,81(0,62-1,06)$ & & $0,85(0,64-1,13)$ & \\
\hline > 4 Salários Mínimos & 27,1 & $0,46(0,27-0,76)$ & & $0,58(0,31-1,05)$ & \\
\hline Atividade Física (min/sem) & & & & & $3,187(0,074)$ \\
\hline Ativo & 45,4 & 1 & $11,479(0,001)$ & 1 & \\
\hline Inativo & 66,4 & $1,49(1,18-1,87)$ & & $1,28(0,98-1,67)$ & \\
\hline \multicolumn{6}{|l|}{ Atividade Física no Passado } \\
\hline Praticou & 40,6 & 1 & $7,322(0,007)$ & 1 & $2,468(0,116)^{2}$ \\
\hline Não Praticou & 58,9 & $1,45(1,11-1,90)$ & & $1,26(0,94-1,69)$ & \\
\hline \multicolumn{6}{|l|}{ Nutrição } \\
\hline Normal & 48,2 & 1 & & 1 & $2,077(0,354)$ \\
\hline Risco de Desnutrição & 68,8 & $1,40(1,08-1,82)$ & $9,310(0,010)$ & $1,21(0,92-1,60)$ & \\
\hline Desnutrição & 86,7 & $1,80(1,06-3,16)$ & & $1,30(0,70-2,41)$ & \\
\hline \multicolumn{6}{|l|}{ Consumo Atual de Alcool } \\
\hline Consome & 43,8 & 1 & $0,252(0,616)$ & & \\
\hline Não Consome & 54,2 & $1,12(0,71-178)$ & & & \\
\hline \multicolumn{6}{|l|}{ Uso do Fumo Atual } \\
\hline Faz Uso & 41,0 & 1 & $0,687(0,407)$ & & \\
\hline Não Faz Uso & 54,2 & $1,24(0,74-2,08)$ & & & \\
\hline \multicolumn{6}{|l|}{ Uso de Medicamentos } \\
\hline Nenhum & 35,7 & 1 & & 1 & $11,177(0,004)^{3}$ \\
\hline 1-2 Medicamentos & 36,7 & $1,03(0,59-1,79)$ & $23,620(<0,001)$ & $0,85(0,46-1,56)$ & \\
\hline$>2$ Medicamentos & 67,9 & $1,90(1,13-3,21)$ & & $1,41(1,13-2,52)$ & \\
\hline \multicolumn{6}{|l|}{ Problemas Cardíacos } \\
\hline Não & 71,1 & 1 & $6,217(0,013)$ & 1 & $0,038(0,845)^{3}$ \\
\hline Sim & 50,0 & $0,70(0,53-0,93)$ & & $0,97(0,71-1,32)$ & \\
\hline \multicolumn{6}{|l|}{ Hipertensão Arterial } \\
\hline Não & 60,1 & 1 & $8,912(0,003)$ & 1 & $4,751(0,029)^{3}$ \\
\hline Sim & 40,2 & $1,67(1,52-2,87)$ & & $1,52(1,43-1,97)$ & \\
\hline \multicolumn{6}{|l|}{ Diabetes Melito } \\
\hline Não & 65,3 & 1 & $4,199(0,040)$ & 1 & $0,000(0,987)^{3}$ \\
\hline Sim & 50,0 & $0,77(0,59-0,99)$ & & $1,00(0,78-1,33)$ & \\
\hline \multicolumn{6}{|l|}{ Déficit Cognitivo (<2) } \\
\hline Sem alterações & 52,1 & 1 & $2,498(0,114)$ & 1 & $0,147(0,702)^{4}$ \\
\hline Com alterações & 68,9 & $1,25(0,95-1,64)$ & & $0,93(0,65-1,33$ & \\
\hline \multicolumn{6}{|l|}{ Sintomatologia Depressiva } \\
\hline Sem & 51,0 & 1 & $3,825(0,050)$ & 1 & $0,033(0,857)^{4}$ \\
\hline Com & 67,4 & $1,33(1,00-178)$ & & $1,03(0,73-1,45)$ & \\
\hline \multicolumn{6}{|l|}{ Capacidade Funcional AIVD } \\
\hline Sem Incapacidade Funcional & 43,9 & 1 & $16,875(<0,001)$ & 1 & $3,673(0,005)^{4}$ \\
\hline Com Incapacidade Funcional & 66,8 & $1,63(1,29-2,07)$ & & $1,36(1,13-1,86)$ & \\
\hline
\end{tabular}


Tabela 3 - Razão de prevalência (RP) bruta e multivariável para varíaveis independentes em relação a percepção negativa da saúde em homens. Brasil, 2008.

Table 3 - Crude and multivariable Prevalence Ratio (PR) for independent variables in the negative perception of health in men. Brazil, 2008.

\begin{tabular}{|c|c|c|c|c|c|}
\hline \multirow[t]{3}{*}{ Variáveis } & \multirow[t]{3}{*}{ Prevalência } & \multicolumn{4}{|c|}{ Percepção Negativa da Saúde } \\
\hline & & \multicolumn{2}{|c|}{ Análise Bruta } & \multicolumn{2}{|c|}{ Análise Multivariável* $^{*}$} \\
\hline & & RP (IC 95\%) & Wald (p-valor) & RP (IC 95\%) & Wald (p-valor) \\
\hline \multicolumn{6}{|l|}{ Cor da Pele } \\
\hline Branca & 44,2 & 1 & $2,166(0,339)$ & & \\
\hline Parda & 39,4 & $0,89(0,62-1,28)$ & & & \\
\hline Negra & 58,3 & $1,32(0,82-2,12)$ & & & \\
\hline Idade (anos) & & $1,04(1,03-1,06)$ & $21,726(<0,001)$ & $1,03(1,01-1,06)$ & $9,133(0,003)^{1}$ \\
\hline \multicolumn{6}{|l|}{ Cidade } \\
\hline Caratinga & 40,7 & 1 & $2,858(0,240)$ & & \\
\hline Ilhéus & 34,7 & $0,85(0,50-1,45)$ & & & \\
\hline Nova Santa Rosa & 51,2 & $1,26(0,90-1,77)$ & & & \\
\hline \multicolumn{6}{|l|}{ Estado Civil } \\
\hline Solteiro & 34,8 & 1 & $12,737(0,005)$ & 1 & $2,221(0,528)^{1}$ \\
\hline Casado & 36,8 & $1,06(0,51-2,18)$ & & $1,25(0,57-2,74)$ & \\
\hline Viúva & 67,6 & $1,94(0,92-4,10)$ & & $1,56(0,69-3,51)$ & \\
\hline Divorciado & 56,3 & $1,62(0,62-4,19)$ & & $1,70(0,63-4,59)$ & \\
\hline \multicolumn{6}{|l|}{ Escolaridade } \\
\hline Analfabeto & 62,9 & 1 & $12,702(0,013)$ & 1 & $2,264(0,687)^{1}$ \\
\hline Primário Incompleto & 47,0 & $0,75(0,51-1,11)$ & & $0,98(0,64-1,50)$ & \\
\hline Primário Completo & 40,4 & $0,64(0,42-0,99)$ & & $0,93(0,57-1,51)$ & \\
\hline Ginasial Completo & 13,0 & $0,21(0,06-0,67)$ & & $0,43(0,13-1,48)$ & \\
\hline Colegial Completo & 12,5 & $0,20(0,05-0,82)$ & & $0,55(0,12-2,51)$ & \\
\hline \multicolumn{6}{|l|}{ Religião } \\
\hline Católico & 42,1 & 1 & $2,153(0,341)$ & & \\
\hline Protestante ou Evangélica & 53,0 & $1,12(0,88-1,80)$ & & & \\
\hline Outros & 38,1 & $0,88(0,52-1,50)$ & & & \\
\hline \multicolumn{6}{|l|}{ Condição Socioeconômica } \\
\hline Classe A/B & 32,0 & 1 & $1,516(0,469)$ & & \\
\hline Classe C & 43,3 & $1,19(0,71-1,99)$ & & & \\
\hline Classe D/E & 50,4 & $1,37(0,81-2,31)$ & & & \\
\hline \multicolumn{6}{|l|}{ Ocupação } \\
\hline Aposentado, mas trabalha & 29,0 & 1 & $2,564(0,633)$ & & \\
\hline Só aposentado & 46,0 & $1,59(0,81-3,11)$ & & & \\
\hline Só dona de casa & 50,0 & $1,72(0,47-6,36)$ & & & \\
\hline Pensionista & 66,7 & $2,30(0,50-10,63)$ & & & \\
\hline Trabalho Remunerado & 36,0 & $1,24(0,49-3,12)$ & & & \\
\hline \multicolumn{6}{|l|}{ Fonte de Renda Atual } \\
\hline Trabalho & 30,6 & 1 & $2,741(0,254)$ & & \\
\hline Previdência /aposentadoria & 45,8 & $1,50(0,88-2,55)$ & & & \\
\hline Outros & 85,7 & $1,96(0,76-5,05)$ & & & \\
\hline
\end{tabular}


Tabela 3 - Razão de prevalência (RP) bruta e multivariável para varíaveis independentes em relação a percepção negativa da saúde em homens. Brasil, 2008. (continuação)

Table 3 - Crude and multivariable Prevalence Ratio (PR) for independent variables in the negative perception of health in men. Brazil, 2008. (continuation)

\begin{tabular}{|c|c|c|c|c|c|}
\hline \multirow[t]{3}{*}{ Variáveis } & \multirow[t]{3}{*}{ Prevalência } & \multicolumn{4}{|c|}{ Percepção Negativa da Saúde } \\
\hline & & \multicolumn{2}{|c|}{ Análise Bruta } & \multicolumn{2}{|c|}{ Análise Multivariável* $^{*}$} \\
\hline & & RP (IC 95\%) & Wald (p-valor) & RP (IC 95\%) & Wald (p-valor) \\
\hline \multicolumn{6}{|l|}{ Rendimento Mensal } \\
\hline Até 2 Salários Mínimos & 49,5 & 1 & $8,122(0,017)$ & 1 & $2,020(0,364)^{1}$ \\
\hline 2-4 Salários Mínimos & 47,9 & $0,97(0,69-1,35)$ & & $0,99(0,70-1,40)$ & \\
\hline > 4 Salários Mínimos & 17,4 & $0,35(0,17-0,73)$ & & $0,57(0,26-1,25)$ & \\
\hline Atividade Física (min/sem) & & & & & $0,771(0,380)^{2}$ \\
\hline Ativo & 35,6 & 1 & $8,682(0,003)$ & 1 & \\
\hline Inativo & 54,8 & $1,16(1,17-2,20)$ & & $1,17(0,82-1,67)$ & \\
\hline \multicolumn{6}{|l|}{ Atividade Física no Passado } \\
\hline Praticou & 39,3 & 1 & $1,074(0,300)$ & & \\
\hline Não Praticou & 46,8 & $1,19(0,86-1,66)$ & & & \\
\hline \multicolumn{6}{|l|}{ Nutrição } \\
\hline Normal & 39,4 & 1 & & 1 & $7,093(0,029)^{2}$ \\
\hline Risco de Desnutrição & 64,3 & $1,72(1,20-2,47)$ & $14,648(0,001)$ & $1,66(1,13-2,43)$ & \\
\hline Desnutrição & 100 & $2,60(1,36-4,98)$ & & $1,46(0,71-2,98)$ & \\
\hline \multicolumn{6}{|l|}{ Consumo Atual de Alcool } \\
\hline Consome & 37,0 & 1 & $0,257(0,612)$ & & \\
\hline Não Consome & 45,3 & $1,13(0,70-1,84)$ & & & \\
\hline \multicolumn{6}{|l|}{ Uso do Fumo Atual } \\
\hline Faz Uso & 43,3 & 1 & $0,001(0,975)$ & & \\
\hline Não Faz Uso & 44,2 & $0,99(0,65-1,53)$ & & & \\
\hline \multicolumn{6}{|l|}{ Uso de Medicamentos } \\
\hline Nenhum & 26,2 & 1 & & 1 & $1,027(0,598)^{3}$ \\
\hline 1-2 Medicamentos & 33,3 & $1,28(0,72-2,25)$ & $16,592(<0,001)$ & $1,03(0,56-1,92)$ & \\
\hline$>2$ Medicamentos & 60,2 & $2,30(1,38-3,85)$ & & $1,26(0,67-2,37)$ & \\
\hline \multicolumn{6}{|l|}{ Problemas Cardíacos } \\
\hline Não & 74,0 & 1 & $18,231(<0,001)$ & 1 & $1,693(0,193)^{3}$ \\
\hline Sim & 36,4 & $0,49(0,36-0,68)$ & & $0,77(0,52-1,14)$ & \\
\hline \multicolumn{6}{|l|}{ Hipertensão Arterial } \\
\hline Não & 53,1 & 1 & $6,974(0,008)$ & 1 & $1,532(0,216)^{3}$ \\
\hline Sim & 34,0 & $0,64(0,46-0,89)$ & & $0,78(0,53-1,15)$ & \\
\hline \multicolumn{6}{|l|}{ Diabetes Melito } \\
\hline Não & 66,1 & 1 & $6,683(0,010)$ & 1 & $1,445(0,229)^{3}$ \\
\hline Sim & 40,6 & $0,62(0,43-0,89)$ & & $0,78(0,52-1,17)$ & \\
\hline \multicolumn{6}{|l|}{ Déficit Cognitivo (<2) } \\
\hline Sem alterações & 41,5 & 1 & $21,494(<0,001)$ & 1 & $0,596(0,440)^{4}$ \\
\hline Com alterações & 81,0 & $2,28(1,61-3,23)$ & & $1,28(0,69-2,38)$ & \\
\hline \multicolumn{6}{|l|}{ Sintomatologia Depressiva } \\
\hline Sem & 44,2 & 1 & $0,010(0,920)$ & 1 & $1,751(0,186)^{4}$ \\
\hline Com & 42,9 & $0,97(0,54-1,75)$ & & $0,60(0,28-1,28)$ & \\
\hline \multicolumn{6}{|l|}{ Capacidade Funcional AIVD } \\
\hline Sem Incapacidade Funcional & 35,4 & 1 & $12,390(<0,001)$ & 1 & $4,738(0,003)^{4}$ \\
\hline Com Incapacidade Funcional & 54,3 & $1,85(1,31-2,60)$ & & $1,79(1,210-1,77)$ & \\
\hline
\end{tabular}


semelhante aos dados para a população brasileira encontrados na Pesquisa Nacional por Amostra de Domicílio, envolvendo 33.515 indivíduos idosos, para as proporções das variáveis sexo, renda, cor da pele, ocupação ${ }^{4}$.

A elevada taxa, correspondente a percepção positiva do estado de saúde, é semelhante à de outros estudos ${ }^{13,14}$. A proporção de sujeitos que classificam o estado de saúde como "excelente/muito boa" ou "boa" apresentou uma média menor de idade do que aqueles que tiveram uma percepção menos otimista da saúde, assim como com relação aos aspectos relacionados a nível baixo de escolaridade e estrato econômico.

Fato interessante foi apresentado em estudo não-probabilístico de um ambulatório de geriatria com 125 sujeitos com idades superiores a 60 anos ${ }^{15}$; nessa investigação foi identificado que o conceito de saúde na velhice se traduz mais por sua condição de autonomia e independência do que pela presença ou ausência de doença. Os autores do estudo ${ }^{15}$ ressaltam que a percepção negativa pode estar relacionada à comparação com outros indivíduos, pois se comparar com outras pessoas tem papel relevante na avaliação e construção da realidade e no enfrentamento de eventos negativos, sobretudo pela capacidade do sujeito de se comparar com pessoas que supostamente estão em condições piores que a sua.

Considerando que a auto-avaliação do estado de saúde reflete, com relativa precisão, o estado geral de saúde, o inquérito populacional realizado em 18 capitais de Estados no Brasil aponta que as piores condições de saúde das regiões brasileiras estão relacionadas às desigualdades sociais ${ }^{16}$.

Entre todos os elementos da situação de vida de uma pessoa idosa, a saúde é o que está mais atrelado a fatores socioeconômicos, à educação e à presença de doenças, fatores que contribuem para determinar a sensação de bem-estar ${ }^{17}$. Os resultados indicam a relação da percepção negativa da saúde com aspectos nutricionais insuficientes, a inatividade física, alterações cognitivas, sintomatologia depressiva e da incapacidade funcional.
Os resultados deste estudo são semelhantes aos de outros estudos ${ }^{13,14,18}$, evidenciando que avaliações subjetivas otimistas não estariam associadas somente à ausência de doenças. Convém ressaltar que a autopercepção negativa de saúde configura-se em preditor de maior risco de mortalidade em comparação com sujeitos que mantêm uma percepção positiva da saúde ${ }^{3}$.

Apesar de a maioria dos idosos ter relatado condições de saúde positiva, quase metade da população estudada encontravase em situação de risco de desnutrição ou até mesmo desnutrida; aproximadamente um terço faz uso de bebidas alcóolicas, fuma regularmente e apresenta sintomas depressivos, e mais da metade relatou alguma incapacidade funcional na realização de atividades instrumentais da vida diária. Estes resultados justificam o impacto do envelhecimento na demanda de serviços de saúde.

A percepção do estado de saúde da pessoa idosa é determinada por vários fatores interatuantes ${ }^{16,17}$. A organização em blocos destas variáveis permite identificar a potencialidade de cada fator de forma independente.

Entre os aspectos intervenientes na percepção de saúde, as alterações decorrentes do declínio das funções cognitivas tem sido identificada como um dos fatores determinantes para a percepção negativa do estado de saúde dos idosos, mesmo quando controlada para variáveis sociodemográficas $^{2}$. Entretanto, a complexidade do processo remete a um constructo explicativo de ciclo, pois o declínio da função cognitiva está relacionado a uma série de fatores, entre os quais se destaca o baixo nível de atividade física ${ }^{19}$.

No presente estudo, na análise não ajustada, diversas variáveis se apresentam associadas à percepção negativa de saúde. Algumas destas também são citadas em outros estudos, a exemplo do aumento da idade que ocasiona muitas vezes uma perda na autoconfiança, refletindo na percepção subjetiva do estado de saúde, nos problemas de saúde e no uso de dois ou mais medicamentos contínuos. 
A percepção da saúde parece estar, em princípio, associada às condições medicamentosas impostas pelas doenças referidas e não ao possível aspecto limitador da doença ao idoso. Neste caso, a sintomatologia depressiva e a incapacidade funcional refletem essas limitações, aparecendo também associadas à percepção menos otimista da saúde ${ }^{3}$.

Fato interessante, na contra-mão deste raciocínio, foi observado em estudo de abordagem qualitativa realizado no município de Bambuí/MG, em que a maior parte dos entrevistados definiu a sua saúde como "boa" ou "razoável," embora a maioria apresentasse condições crônicas de saúde que variavam em grau e necessidade ${ }^{1}$. Em geral, os idosos condicionam o significado de saúde e de doença à capacidade laboral ou de realização de atividades de lazer, o que sugere uma forte relação entre a percepção positiva ou negativa de saúde às condições de realizar atividades da vida diária e de interação social ${ }^{1}$.

$\mathrm{Na}$ análise multivariada poucas variáveis permaneceram associadas à percepção negativa da saúde, em função das possíveis interações e confusões. Sendo assim, para as mulheres apenas o uso de dois ou mais medicamentos, a hipertensão arterial e a incapacidade funcional na realização das atividades instrumentais da vida diária permaneceram associados à avaliação negativa da saúde.

A presença da hipertensão arterial no modelo final de estudo pode ser explicada, em parte, pelo impacto da enfermidade no dia-a-dia das pessoas, exigindo o controle pressórico e o uso regular de medicamentos. Como o uso contínuo de medicamentos é um indicador para o próprio indivíduo que algo não está adequado, aliado as dificuldades ao acesso e controle no uso diário do medicamento, pode interferir para uma percepção menos otimista da saúde.

Nos homens, o aumento da idade, a desnutrição e a incapacidade funcional foram as variáveis que permaneceram no modelo de análise ajustada. $\mathrm{O}$ aumento da idade tem sido constantemente associado à perda da auto-eficácia e à avaliação negativa da saúde. Neste caso, a surpresa foi o fato da idade não ter permanecido no modelo final para o sexo feminino, talvez por conta da força de outras variáveis que se sobrepuseram aos aspectos demográficos.

Já o estado de desnutrição é algo que merece atenção, principalmente quando se avalia os homens. Talvez parte desta insatisfação esteja atrelada ao sentimento de incapacidade funcional e de receio com relação às mudanças corporais frente à desnutrição. Entretanto, na maior parte das vezes o estado de desnutrição está atrelado à baixa condição econômica e a uma maior vulnerabilidade para ser acometido por doenças.

A incapacidade funcional reflete a condição de realizar as funções sociais, sendo que alguns resultados indicam que a auto-avaliação positiva de saúde tende a refletir certo esforço do idoso em permanecer ativo, preservar sua identidade intersubjetiva e o seu papel no grupo ${ }^{1,4}$.

É interessante destacar que as condições físicas não são suficientes para determinar a forma de o idoso perceber o estado de saúde. Entretanto, algumas condições patológicas e comportamentais contribuem para uma percepção negativa do estado de saúde.

Algumas limitações podem ser destacadas no estudo: o fato de os idosos disponíveis para entrevistas serem aqueles supostamente aqueles com percepção de saúde positiva; e o delineamento transversal impede assegurar a relação de causa e efeito entre as variáveis, sendo que as medidas de autopercepção podem ter sofrido interferência em relação à baixa escolaridade dos participantes e de aspectos motivacionais dos participantes. Entretanto, o procedimento estatístico adotado permitiu estimar as prevalências das variáveis associadas à percepção negativa de saúde; além disso, os avaliadores do estudo passaram por treinamento, no intuito de minimizarem as interferências motivacionais e até mesmo de sincronizar as explicações durante a entrevista às possíveis dúvidas dos respondentes em face à variação de escolaridade. 


\section{Conclusões}

A presença de limitações na realização das atividades instrumentais da vida diária, mesmo quando controladas para os fatores socioeconômicos, demográficos e de saúde, manteve-se associada à percepção negativa do estado de saúde. Desta forma, o elemento essencial mediador da auto-avaliação pessimista da saúde pode ser a própria dependência, sugerindo que o declínio na capacidade funcional possa ser o maior indicador para o próprio indivíduo de sua condição na velhice.

A elaboração de políticas públicas direcionadas a saúde e ao bem estar da pessoa idosa exige o constante monitoramento de diversos comportamentos e condições que possam afetar o idoso no seu dia-a-dia. Os resultados desse estudo oferece ao gestor de saúde informações que podem auxiliar a redução de custos e tempo na avaliação dos idosos.

\section{Referências}

1. Fonseca MGUP, Firmo JOA, Loyola Filho, AI; Uchôa, E. Papel da autonomia na auto-avaliação da saúde do idoso. Rev Saúde Pública 2010; 44: 159-65.

2. Freitas DHM, Campos FCA, Linhares LQ, Santos CR, Ferreira CB, Diniz BS, Tavares A. Autopercepção de saúde e desempenho cognitivo em idosos residentes na comunidade. Rev Psiq Clin 2010; 37: 32-5.

3. Desalvo KB, Muntner P. Discordance between physician and patient self-rated health and all-cause mortality. Ochsner J 2011; 11: 232-40.

4. Alves LC, Leite IC, Machado CJ. Fatores Associados à incapacidade funcional dos idosos no Brasil: análise multinível. Rev Saúde Pública 2010; 44: 1-11.

5. Kivinen P, Halonen P, Eronen M, Nissinen A. Self-rated health, physician-rated health and associated factors among elderly men: The Finnish cohorts of the Seven Countries Study. Age Aging 1998; 27: 41-7.

6. Leblanc ES, Wang PY, Lee CG, Barrett-Connor E, Cauley JA, Hoffman AR et al. Higher testosterone levels are associated with less loss of lean body mass in older men. J Clin Endocrinol Metab 2011; 96(12): 3855-63.

7. Arango HG. Bioestatística teórica e computacional com banco de dados reais em disco. Rio de Janeiro: Guanabara Koogan; 2005.

8. Organização Mundial da Saúde. Classificação Internacional de Doenças e problemas relacionados à saúde: décima revisão (CID-10). São Paulo: Centro Colaborador da OMS para Classificação de Doenças em Português; 1993

9. Guigoz Y, Vellas B. The Mini Nutritional Assessment (MNA) for grading the nutritional state of elderly patients: presentation of the MNA, history and validation. Nestle Nutr Workshop Ser Clin Perfrom Programme 1999; 1: 3-11.

10. Santos RL, Virtuoso Júnior JS. Confiabilidade da versão Brasileira da escala de atividades instrumentais da vida diária. RBPS 2008; 21: 290-6.
11. Almeida OP, Almeida SA. Confiabilidade da versão brasileira da escala de depressão em geriatria (GDS) versão reduzida. Arq Neuropsiquiatr 1999; 57: 421-6.

12. Benedetti TRB, Mazo GZ, Barros MVG. Aplicação do questionário internacional de atividades físicas (IPAQ) para avaliação do nível de atividades físicas de mulheres idosas: validade concorrente e reprodutibilidade testereteste. Rev Bras Ciênc Mov 2004; 12: 25-34.

13. Ramos LR. Fatores determinantes do envelhecimento saudável em idosos residentes em centro urbano: Projeto Epidoso, São Paulo. Cad Saude Publica 2003; 19: 793-8.

14. Virtuoso Júnior JS, Guerra RO. Fatores associados às limitações funcionais em idosas de baixa renda. Rev Assoc Med Bras 2008; 54: 430-5.

15. Spolito G; Diogo MJD; Cintra FA; Neri AL; Guariento ME; Sousa MLR. Relações entre o bem-estar subjetivo e a funcionalidade em idosos em seguimento ambulatorial. Rev Bras Fisioter 2010; 14: 81-9.

16. Souza MC, Otero UB, Almeida LM, Turci SRB, Figueiredo VC, Lozan JA. Auto-avaliação de saúde e limitações físicas decorrentes de problemas de saúde. Rev Saúde Pública 2008; 42(4): 741-9.

17. Silva MCS, Lautert L. O senso de auto-eficácia na manutenção dos comportamentos promotores de saúde de idosos. Rev Esc Enferm USP. 2010; 44: 61-7.

18. Ruiz T, Chalita LVAS, Barros MBA. Estudo de sobrevivência de uma coorte de pessoas de 60 anos e mais no município de Botucatu (SP) - Brasil. Rev bras epidemiol 2003; 6: 227-36.

19. Vance D, Wadley V, Ball K, Roenker D, Rizzo M. The effects of physical activity and sedentary behavior on cognitive health in older people. J Aging Phys Act 2005; 13(3): 294-313.

Recebido em: 14/02/11

Versão final apresentada em: 27/12/11 Aprovado em: 03/02/12 
Errata

Correction
Prevalência e fatores associados à percepção negativa da saúde em pessoas idosas no Brasil

Prevalence and factors associated with negative health

perception by the Brazilian elderly

Volume 15, número 1, março/2012, Página 49:

Onde se lê:

- Aldemis Smith-Menezes

O correto é:

- Aldemir Smith-Menezes 